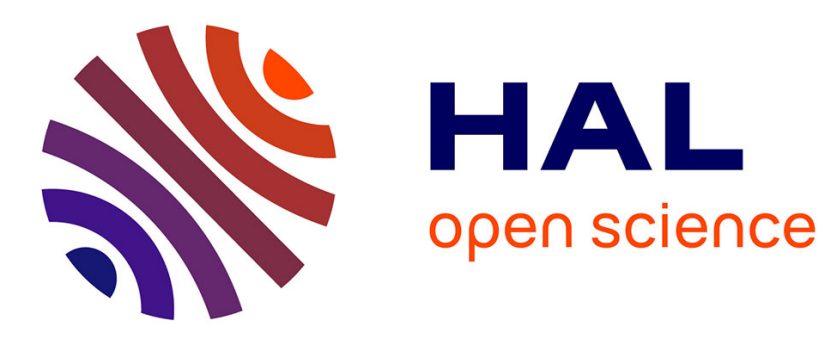

\title{
A homogenization model of the Voigt type for skeletal muscle
}

\author{
L.A. Spyrou, M. Agoras, Kostas Danas
}

\section{To cite this version:}

L.A. Spyrou, M. Agoras, Kostas Danas. A homogenization model of the Voigt type for skeletal muscle. Journal of Theoretical Biology, 2017, 414, pp.50-61. 10.1016/j.jtbi.2016.11.018 . hal-01627518

\section{HAL Id: hal-01627518 \\ https://hal-polytechnique.archives-ouvertes.fr/hal-01627518}

Submitted on 21 Dec 2017

HAL is a multi-disciplinary open access archive for the deposit and dissemination of scientific research documents, whether they are published or not. The documents may come from teaching and research institutions in France or abroad, or from public or private research centers.
L'archive ouverte pluridisciplinaire HAL, est destinée au dépôt et à la diffusion de documents scientifiques de niveau recherche, publiés ou non, émanant des établissements d'enseignement et de recherche français ou étrangers, des laboratoires publics ou privés. 


\section{A homogenization model of the Voigt type for skeletal muscle}

Article in Journal of Theoretical Biology · November 2016

DOI: 10.1016/j.jtbi.2016.11.018

CITATIONS

0

3 authors:

\section{Leonidas A Spyrou}

The Centre for Research and Technology, Hel...

8 PUBLICATIONS 40 CITATIONS

SEE PROFILE

Kostas Danas

École Polytechnique

44 PUBLICATIONS 622 CITATIONS

SEE PROFILE

\section{Michalis Agoras}

University of Thessaly

11 PUBLICATIONS 166 CITATIONS

SEE PROFILE 


\section{Author's Accepted Manuscript}

A homogenization model of the Voigt type for skeletal muscle

L.A. Spyrou, M. Agoras, K. Danas

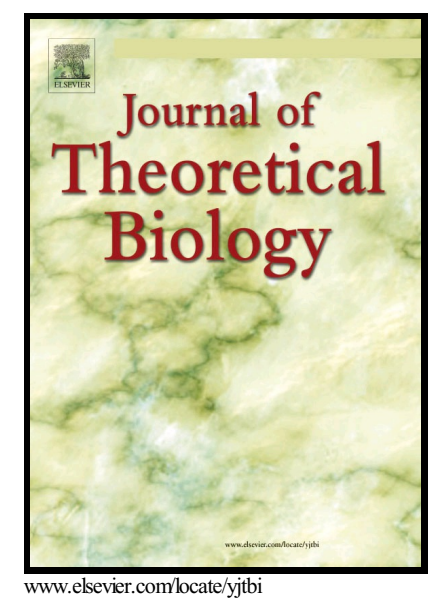

PII: $\quad$ S0022-5193(16)30383-6

DOI: $\quad$ http://dx.doi.org/10.1016/j.jtbi.2016.11.018

Reference: YJTBI8871

To appear in: Journal of Theoretical Biology

Received date: 28 July 2016

Revised date: 12 November 2016

Accepted date: 18 November 2016

Cite this article as: L.A. Spyrou, M. Agoras and K. Danas, A homogenizatior model of the Voigt type for skeletal muscle, Journal of Theoretical Biology http://dx.doi.org/10.1016/j.jtbi.2016.11.018

This is a PDF file of an unedited manuscript that has been accepted fo publication. As a service to our customers we are providing this early version o the manuscript. The manuscript will undergo copyediting, typesetting, an review of the resulting galley proof before it is published in its final citable form Please note that during the production process errors may be discovered whic could affect the content, and all legal disclaimers that apply to the journal pertain 


\title{
A homogenization model of the Voigt type for skeletal muscle
}

\author{
L.A. Spyrou ${ }^{\mathrm{a}, *}$, M. Agoras ${ }^{\mathrm{b}}$, K. Danas ${ }^{\mathrm{c}}$ \\ ${ }^{a}$ Institute for Research 8 Technology - Thessaly, Centre for Research 63 Technology Hellas (CERTH), 38333 Volos, Greece \\ ${ }^{b}$ Department of Mechanical Engineering, University of Thessaly, 38334 Volos, Greece \\ ${ }^{c}$ LMS, CNRS, École Polytechnique, Université Paris-Saclay, 91128 Palaiseau, France
}

\begin{abstract}
A three-dimensional constitutive model for skeletal muscle incorporating microstructural characteristics is developed and numerically implemented in a general purpose finite element program. The proposed model takes into account explicitly the volume fractions of muscle fibers and connective tissue by using the Voigt homogenization approach to bridge the different length scales of the muscle structure. The model is used to estimate the active and passive homogenized muscle response. Next, the model is validated by experimental data and periodic three-dimensional unit cell calculations comprising various fiber volume fractions and mechanical properties of the constituents. The model is found to be in very good agreement with both the experimental data and the finite element results for all the examined cases. The influence of fiber volume fraction and material properties of constituents on effective muscle response under several loading conditions is examined.
\end{abstract}

Keywords: muscle tissue, intramuscular connective tissue, muscle fiber, constitutive modeling, unit-cell, finite element analysis

\section{Introduction}

Skeletal muscle is a complex hierarchical structure consisting of force-producing fibers and connective tissue. Each muscle fiber is surrounded by endomysium and a group of muscle fibers is surrounded by perimysium to form a muscle fascicle. The entire muscle volume is wrapped in an epimysial connective tissue covering. Although the intramuscular connective tissue (endomysium, perimysium, epimysium) accounts for only $1-10 \%$ of a healthy skeletal muscle volume (Kjær, 2004), several studies have shown that it plays an important role in the muscle's structural and functional characteristics, i.e. a) it adds to the passive elastic response of the muscle (Trotter et al., 1995), b) it has a major contribution to myo-fascial force transmission between neighboring fibers and fascicles (Huijing, 1999), c) it is implicated in muscle diseases (e.g. muscular dystrophy (Emery et al., 2002; Mann et al., 2011), spasticity (Lieber et al., 2003)), and

\footnotetext{
* Corresponding author.

Email addresses: 1spyrou@ireteth.certh.gr (L.A. Spyrou), agoras@mie.uth.gr (M. Agoras), kdanas@lms.polytechnique.fr (K. Danas) 
immobilization (Jozsa et al., 1988), which certainly cause significant alterations in the volume fractions and/or the mechanical properties of the muscle constituents. Thus, both muscle fibers and connective tissue determine the overall properties of the whole muscle through their microstructural organization and functional cooperation.

Most available computational models of skeletal muscle have simplified the muscle structure and behavior into a 1D lumped-parameter description of whole muscle contraction (e.g. Zajac, 1989) in order to mainly study muscle-driven whole body movement. More recently, towards investigating complex structural and functional aspects of skeletal muscle, three-dimensional tissue-level constitutive models were developed based on the principles of continuum mechanics where muscle tissue is represented as a fiber-reinforced composite (e.g. Blemker et al., 2005; Ehret et al., 2011; Johansson et al., 2000; Lemos et al., 2004; Liang et al., 2006; Martins et al., 1998; Röhrle and Pullan, 2007; Spyrou and Aravas, 2011). These models have led to a better understanding of force distributions, internal tissue loadings, and shape deformations of specific muscle structures (e.g. biceps brachii (Blemker et al., 2005), tibialis anterior (Lemos et al., 2004), masseter (Röhrle and Pullan, 2007), ankle plantar flexors (Spyrou and Aravas, 2012), sartorius (Böl and Reese, 2008)) in the context of finite element analysis. Some attempts have also been made to include electrophysiological aspects of skeletal muscle in the constitutive description of the material's behavior (Böl et al., 2011; Heidlauf and Röhrle, 2014; Hernández-Gascón et al., 2013).

However, these models do not explicitly include any microstructural information associated with intramuscular connective tissue, such as geometry, mechanical properties, and volume fraction in the muscle. Thus, new constitutive models have to be developed in order to provide insights into skeletal muscle's macroscopic behavior upon tissue's microstructural changes. Since these structural changes are mainly associated with skeletal muscle diseases, such muscle models become more important because they may lead to a better understanding of diseased muscles' mechanical behavior. Only recently, microstructural models have been developed to assess hypoxic damage in skeletal muscles (Ceelen et al., 2008) and estimate the macroscopic along-fiber shear modulus of healthy (Sharafi and Blemker, 2010) and diseased (Virgilio et al., 2015) muscles. While each one of these studies has its own significant contribution to the understanding of the effective response of skeletal muscles, none of them leads to a tissue-level constitutive model of muscle that explicitly includes microstructural information and which can then be used to investigate microstructural effects on the macroscopic behavior of 3D anatomical muscle structures.

In this work, we propose a simple homogenization model for the macroscopic response of skeletal muscle along with an algorithm for its numerical implementation in a general purpose finite element program. The macroscopic (homogenized) behavior of the skeletal muscle is obtained by employing the Voigt hypothesis that the strain field in the composite is uniform. Nonetheless, the present analytical and numerical models are expected to shed light on how the macroscopic response of the skeletal muscle is affected by its micromechanical and microstructural properties. More specifically, in Section 2 an analytical constitutive model for 
skeletal muscle that takes into account the volume fractions and the material properties of its constituents (muscle fibers and connective tissue) is introduced. Subsequently, Section 3 presents in detail a finite element (FE) periodic unit-cell framework which will be used to calculate the homogenized muscle response numerically. In Section 4 comparisons are performed between the analytical model predictions, experimental data and the FE results under various loading conditions. Also, we conduct a number of parametric studies in order to further investigate muscle's macroscopic response upon micromechanical changes. The results of the study and the main features of the model are discussed in Section 5. Finally, we conclude in Section 6 .

\section{The analytical model}

Skeletal muscles may be viewed as composite materials consisting of muscle fibers embedded in intramuscular connective tissue. Typically, the fibers are approximately aligned and distributed in the transverse plane at three well-separated length scales. The connective tissue surrounding the fibers at the lowest, intermediate and highest length scale is referred to as the endomysium, perimysium, and epimysium, respectively. These three types of tissue are also known collectively as the extracellular matrix (ECM).

In this work, we model skeletal muscle as a two-phase composite material made out of a large number of homogeneous muscle fibers (phase 1) surrounded by and perfectly bonded to a homogeneous ECM (phase 2). The fibers are further assumed to be aligned along a given direction $\mathbf{m}_{0}$ in the undeformed configuration. The details concerning the constitutive relations characterizing the material behavior of the constituent phases are given in subsection 2.1, while the homogenization procedure used to obtain the macroscopic or homogenized behavior of the composite skeletal muscle is discussed in subsection 2.2 .

\subsection{Constitutive relations for the phases}

Following earlier work (e.g. Sharafi and Blemker, 2010, 2011; Virgilio et al., 2015), we model the ECM and the muscle fibers as two different, nearly incompressible, transversely isotropic solids, characterized by the same symmetry axis $\mathbf{m}_{0}$ in the undeformed configuration. In particular, we assume that the total true stress tensor $\boldsymbol{\sigma}^{(r)}$ at any given material point $\mathbf{X}$ in the continuum may be written as the sum of an isotropic part $\boldsymbol{\sigma}_{i}^{(r)}$ and an anisotropic part $\boldsymbol{\sigma}_{a}^{(r)}$ :

$$
\boldsymbol{\sigma}^{(r)}=\boldsymbol{\sigma}_{i}^{(r)}+\boldsymbol{\sigma}_{a}^{(r)}
$$

where the superscript $r=1$ when $\mathbf{X}$ is in the fiber phase and $r=2$ when $\mathbf{X}$ is in the ECM phase. In connection with the above decomposition (1) of the stress tensor, we remark that $\boldsymbol{\sigma}_{a}^{(r)}$ represents the stress response in the material's preferred direction, and $\boldsymbol{\sigma}_{i}^{(r)}$ is associated with the non-fibrous matrix and biofluids surrounding the fibrous part of the constituents and mainly represents any response under shear loading and transverse normal straining with respect to the material's preferred direction. Next, we provide specific 
constitutive equations for $\boldsymbol{\sigma}_{i}^{(r)}$ and $\boldsymbol{\sigma}_{a}^{(r)}$. It should also be remarked, however, that the homogenization estimates obtained in subsection 2.2 hold for completely general constitutive relations for the phases.

The isotropic part of the stress $\boldsymbol{\sigma}_{i}^{(r)}$ in (1) is assumed to be of the following hyperelastic form

$$
\boldsymbol{\sigma}_{i}^{(r)}=\frac{2}{J} \boldsymbol{F} \frac{\partial W^{(r)}}{\partial \boldsymbol{C}} \boldsymbol{F}^{T}
$$

where $\boldsymbol{F}$ stands for the deformation gradient at point $\mathbf{X}, J=\operatorname{det} \boldsymbol{F}, \boldsymbol{C}=\boldsymbol{F}^{T} \boldsymbol{F}$ is the right Cauchy-Green deformation tensor and $W^{(r)}$ is the associated stored energy function of phase $r$. The latter function is taken to be of the following Neo-Hookean form

$$
W^{(r)}(\boldsymbol{F})=\frac{G^{(r)}}{2}\left(\bar{I}_{1}-3\right)+\frac{K^{(r)}}{2}(J-1)^{2},
$$

where $\bar{I}_{1}=J^{-2 / 3} I_{1}$, with $I_{1}=\operatorname{tr} \boldsymbol{C}$, while the constants $G^{(r)}$ and $K^{(r)}$ are respectively the shear and bulk moduli of phase $r$. Making use of expression (3) in (2), it can be easily shown that

$$
\boldsymbol{\sigma}_{i}^{(r)}=\frac{G^{(r)}}{J}\left(\bar{B}-\frac{1}{3} \operatorname{tr}(\bar{B}) \boldsymbol{\delta}\right)+K^{(r)}(J-1) \boldsymbol{\delta}
$$

where $\overline{\boldsymbol{B}}=J^{-2 / 3} \boldsymbol{B}$, with $\boldsymbol{B}=\boldsymbol{F} \boldsymbol{F}^{T}$ being the left Cauchy-Green deformation tensor, and $\boldsymbol{\delta}$ is the secondorder identity tensor. Note that, incompressible Neo-Hookean behavior corresponds to the limiting case $K^{(r)} \longrightarrow \infty$, along with the constraint $J=1$, in (3) and (4). The above Neo-Hookean relations are commonly used to model the isotropic behavior of soft biological tissues (e.g. Holzapfel et al., 2000).

The anisotropic part of the stress $\boldsymbol{\sigma}_{a}^{(r)}$ in (1) is assumed to be of the form (Liang et al., 2006; Spyrou and Aravas, 2011)

$$
\boldsymbol{\sigma}_{a}^{(r)}=\sigma^{(r)} \mathbf{m} \mathbf{m}
$$

where

$$
\mathbf{m}=\frac{1}{\left|\boldsymbol{F} \cdot \mathbf{m}_{0}\right|} \boldsymbol{F} \cdot \mathbf{m}_{0}
$$

is the unit vector along the symmetry axis of transverse isotropy in the deformed configuration, and $\sigma^{(r)}$ is the current axial stress, which is given in terms of the reference axial stress $\sigma_{0}^{(r)}$ by

$$
\sigma^{(r)}=\sigma_{0}^{(r)} \frac{A_{0}}{A}=\sigma_{0}^{(r)} \frac{d s}{d s_{0}}=\sigma_{0}^{(r)} \lambda_{m}=\sigma_{0}^{(r)}\left(1+\varepsilon_{0}^{m}\right)
$$

where $A$ and $A_{0}$ denote respectively the current and reference material cross-sectional areas normal to the fiber direction, $\lambda_{m}$ is the axial stretch ratio in the muscle fiber direction and $\varepsilon_{0}^{m}=\lambda_{m}-1$ is the nominal axial strain in the muscle fiber direction. Note that, in (7) use has been made of the incompressibility constraint $A_{0} d s_{0}=A d s$. The constitutive relation for $\sigma_{0}^{(r)}$ is different for the muscle fibers $(r=1)$ and the $\operatorname{ECM}(r=2)$, and it is defined separately in the following subsections. 


\subsubsection{Anisotropic stress $\sigma_{0}^{(1)}$ for the muscle fibers}

Muscle fibers exhibit both "active" and "passive" mechanical characteristics. Thus, the nominal longitudinal stress in a muscle fiber can be written as the sum of an active and a passive part:

$$
\sigma_{0}^{(1)}=\sigma_{0}^{(1, a c t)}+\sigma_{0}^{(1, p a s)} .
$$

The active behavior is known to depend on the fiber length and velocity of contraction as well as on the activation level of the muscle, whereas the passive behavior depends mainly on the fiber length. From a "continuum" point of view, this means that the muscle fiber stress depends on the local strain, strain-rate, and activation level. Following Van Leeuwen and Kier (1997), we write

$$
\sigma_{0}^{(1)}=\sigma_{\max } f_{a} f_{e}\left(\varepsilon_{0}^{m}\right) f_{r}\left(\dot{\varepsilon}_{0}^{m}\right)+f_{p}^{(1)}\left(\varepsilon_{0}^{m}\right)
$$

where $\sigma_{\max }$ is the maximum isometric stress that appears at optimal fiber length, $f_{a}$ is the activation function that controls the magnitude of $\sigma_{0}^{(1, a c t)}, f_{e}$ describes the dependence of the active stress on the nominal longitudinal strain $\varepsilon_{0}^{m}, f_{r}$ is the function that relates the active muscle stress to the nominal longitudinal strain rate $\dot{\varepsilon}_{0}^{m}$, and $f_{p}^{(1)}$ describes the dependence of the passive stress on the nominal longitudinal strain $\varepsilon_{0}^{m}$. In the above equation functions $\left(f_{a}, f_{e}, f_{r}\right)$ are dimensionless and normalized so that

$$
0 \leq f_{a} \leq 1, \quad \max _{\varepsilon_{0}^{m}} f_{e}\left(\varepsilon_{0}^{m}\right)=1, \quad f_{r}(0)=1
$$

In this work, the active stress-strain function $f_{e}$ is described by a normalized Weibull distribution with two parameters $\varepsilon_{0}^{m, m i n}$ and $\varepsilon_{0}^{m, o p t}$ as proposed in Ehret et al. (2011)

$$
f_{e}\left(\varepsilon_{0}^{m}\right)= \begin{cases}\frac{b_{1}}{b_{2}} \exp \left[\frac{\left(b_{1}+b_{2}\right)\left(b_{2}-b_{1}\right)}{2 b_{2}{ }^{2}}\right] & \text { if } b_{1}<0 \\ 0 & \text { else }\end{cases}
$$

where $b_{1}=\varepsilon_{0}^{m, m i n}-\varepsilon_{0}^{m}, b_{2}=\varepsilon_{0}^{m, m i n}-\varepsilon_{0}^{m, o p t}, \varepsilon_{0}^{m, m i n}$ denotes the minimum nominal fiber strain at which muscle fiber begins to develop force, and $\varepsilon_{0}^{m, o p t}$ is the nominal optimal fiber strain, i.e. the fiber strain at which muscle fiber reaches optimal length.

The well-known hyperbolic function $f_{r}$ is used to describe the strain rate dependence on the muscle fiber stress (Böl and Reese, 2008; Van Leeuwen and Kier, 1997)

$$
f_{r}\left(\dot{\varepsilon}_{0}^{m}\right)= \begin{cases}d-(d-1) \frac{1+t^{*}}{1-k_{c} k_{e} t^{*}} & \text { if } t^{*}<0 \\ \frac{1-t^{*}}{1+k_{c} t^{*}} & \text { if } t^{*} \geq 0\end{cases}
$$

where $t^{*}=\frac{\dot{\varepsilon}_{0}^{m}}{\dot{\varepsilon}_{0}^{m, \min }}, \dot{\varepsilon}_{0}^{m, \text { min }}$ is the minimum nominal strain rate, and $k_{c}, k_{e}, d$ are dimensionless material constants. 
Recent experimental studies have shown a linear stress-strain behavior of single muscle fibers (Meyer and Lieber, 2011; Smith et al., 2011), thus the muscle fiber passive stress-strain relation $f_{p}^{(1)}$ can be described by a linear function of nominal fiber strain as

$$
f_{p}^{(1)}\left(\varepsilon_{0}^{m}\right)= \begin{cases}E_{p}\left(\varepsilon_{0}^{m}-\varepsilon_{0}^{m, o p t}\right) & \text { if } \varepsilon_{0}^{m}>\varepsilon_{0}^{m, o p t} \\ 0 & \text { else }\end{cases}
$$

where we take into account that $\varepsilon_{0}^{m, o p t}$ is the minimum strain at which a muscle fiber develops passive stress (Blemker et al., 2005; Zajac, 1989) and $E_{p}$ is the muscle fiber's passive elastic modulus.

\subsubsection{Anisotropic stress $\sigma_{0}^{(2)}$ for the $E C M$}

The ECM's collagenous fiber network develops only "passive" stresses and, in accord with experimental studies, microscopically possesses a preferred direction. In particular, the orientation distribution of collagen fibrils in endomysium shows a circumferential bias with a mean fibril angle of about $60^{\circ}$ relative to the muscle fiber direction (Purslow and Trotter, 1994). Accordingly, the collagen fibrils in perimysium are arranged in cross plies and oriented at about $\pm 55^{\circ}$ with respect to the muscle fiber direction (Purslow, 1989; Purslow and Trotter, 1994).

In this study we consider the helical structure of ECM's collagen fibrils surrounding the muscle fibers similarly with the modeling scheme followed in Gindre et al. (2013); it is assumed that ECM's fiber network represents a helical fiber distribution with a preferred angle wrapped around the fibers. Also, we take into account that the collagen fibril helices are constrained to deform with the muscle fibers so that the stretch in a collagen helix $\lambda_{H}$ can be expressed in terms of the stretch $\lambda_{m}$ and the initial collagen fibril angle with respect to the muscle fiber $\theta_{0}$ (Gindre et al., 2013) as

$$
\lambda_{H}=\sqrt{\frac{1+\lambda_{m}{ }^{3} \tan ^{2}\left(\frac{\pi}{2}-\theta_{0}\right)}{\lambda_{m}\left(1+\tan ^{2}\left(\frac{\pi}{2}-\theta_{0}\right)\right)}}, \quad \lambda_{m}=\sqrt{\mathbf{m}_{0} \cdot \boldsymbol{C} \cdot \mathbf{m}_{0}} .
$$

Therefore, the total nominal fiber stress of ECM can be written as a function of the stretch in the muscle fiber direction and the declination angle between the collagen fibrils and the muscle fiber

$$
\sigma_{0}^{(2)}=f_{p}^{(2)}\left(\lambda_{H}\right)=f_{p}^{(2)}\left(\lambda_{m}, \theta_{0}\right)
$$

An exponential function $f_{p}^{(2)}$ is adopted here to describe the mechanical behavior of the collagenous connective tissue (Holzapfel et al., 2000)

$$
f_{p}^{(2)}\left(\lambda_{H}\right)= \begin{cases}A 1\left(e^{A 2\left(\lambda_{H}-1\right)}-1\right) & \text { if } \lambda_{H}>1 \\ 0 & \text { else }\end{cases}
$$

where $A 1$ and $A 2$ are material coefficients. In deriving the last equation we also took into account that fibers (both ECM's collagen fibers and muscle fibers) do not support passive compressive loads as often assumed in applications of continuum mechanics to soft fibrous materials (e.g. Holzapfel and Ogden, 2015). 


\subsection{Homogenization estimates for the macroscopic behavior}

Consider a representative volume element (RVE) of composite skeletal muscle material, as defined above, which in the undeformed configuration occupies a region $V_{0}$ with boundary $\partial V_{0}$, and let $V_{0}^{(1)}$ and $V_{0}^{(2)}$ be the complementary parts of $V_{0}$ occupied by muscle fibers (phase 1) and ECM (phase 2), respectively. Then, the volume fractions of the muscle fibers and the ECM in the RVE are

$$
\frac{\left|V_{0}^{(1)}\right|}{\left|V_{0}\right|} \equiv c, \quad \frac{\left|V_{0}^{(2)}\right|}{\left|V_{0}\right|} \equiv 1-c,
$$

respectively. The particular shape of $\partial V_{0}$ is not important for our purpose here. We assume, however, that the characteristic length of $V_{0}$ is much larger than the diameter of a typical fiber, so that the notion of the homogenized behavior of the composite at the level of the RVE makes sense.

The homogenization problem for the composite material of interest may be defined by means of the following boundary condition

$$
\mathbf{x}(t)=\widehat{\boldsymbol{F}}(t) \mathbf{X} \quad \forall \quad \mathbf{X} \quad \in \quad \partial V_{0}
$$

where $\mathbf{X}$ and $\mathbf{x}(t)$ denote the position vectors of any given material point on the boundary of the body in the undeformed and deformed (at time $t$ ) configuration, respectively, while $\widehat{\boldsymbol{F}}(t)$ is a deformation gradient tensor which may vary with time $t$, but is independent of $\mathbf{X}$, i.e., $\widehat{\boldsymbol{F}}(t)$ is constant for given $t$. Note that, due to the assumed incompressibility of the material, the volume fractions of the phases (17) are independent of the deformation of the body. Letting $\boldsymbol{F}(\mathbf{X}, t)$ be the (unknown) deformation gradient field produced in the RVE under the boundary conditions (18), it can be easily shown that

$$
\frac{1}{\left|V_{0}\right|} \int_{V_{0}} \boldsymbol{F}(\mathbf{X}, t) \mathrm{d} \mathbf{X}=\widehat{\boldsymbol{F}}(t)
$$

i.e., the average of $\boldsymbol{F}(\mathbf{X}, t)$ over the RVE $V_{0}$ is equal to the applied deformation gradient $\widehat{\boldsymbol{F}}(t)$. For convenience and latter reference, we also introduce at this point the notation

$$
\widehat{\boldsymbol{\sigma}}(t) \equiv \frac{1}{|V|} \int_{V} \boldsymbol{\sigma}(\mathbf{x}, t) \mathrm{d} \mathbf{x}
$$

for the average of the corresponding (unknown) true stress field $\boldsymbol{\sigma}(\mathbf{x}, t)$ over the region $V$ occupied by the body in the deformed (at time $t$ ) configuration.

Under the above considerations, the macroscopic or homogenized behavior of the skeletal muscle is defined by the relation between the average stress $\widehat{\boldsymbol{\sigma}}(t)$ and the history of the average deformation of the RVE, as prescribed by the deformation gradient $\widehat{\boldsymbol{F}}(t)$. It is important to realize, however, that the determination of this relation requires the computation of the local stress and deformation fields developed in the RVE under the boundary conditions (18), which is in general a very difficult problem. In order to handle this difficulty, in this work we make use of the Voigt approximation that the deformation gradient field $\boldsymbol{F}(\mathbf{X}, t)$ is uniform in the RVE and, therefore, equal to $\widehat{\boldsymbol{F}}(t)$, i.e.,

$$
\boldsymbol{F}(\mathbf{X}, t) \approx \widehat{\boldsymbol{F}}(t),
$$


which implies, in turn, that the stress field $\boldsymbol{\sigma}(\mathbf{x}, t)$ is constant-per-phase. Thus, the approximation (21) yields the following estimate for the homogenized behavior of the skeletal muscle

$$
\widehat{\boldsymbol{\sigma}}(t) \approx c \boldsymbol{\sigma}^{(1)}(\widehat{\mathbf{F}}(t))+(1-c) \boldsymbol{\sigma}^{(2)}(\widehat{\mathbf{F}}(t))
$$

where the notation $\boldsymbol{\sigma}^{(r)}(\widehat{\boldsymbol{F}}(t))$, with $r=1,2$, has been used in order to emphasize the fact that the response functions $\boldsymbol{\sigma}^{(r)}$ of the phases, defined in the previous subsection, must be evaluated at $\boldsymbol{F}=\widehat{\boldsymbol{F}}(t)$.

Taking into account the decomposition (1) for $\boldsymbol{\sigma}^{(r)}$, the macroscopic constitutive relation (22) may also be written as a sum of an isotropic part $\widehat{\boldsymbol{\sigma}}_{i}(t)$ and an anisotropic part $\widehat{\boldsymbol{\sigma}}_{a}(t)$ :

$$
\widehat{\boldsymbol{\sigma}}(t)=\widehat{\boldsymbol{\sigma}}_{i}(t)+\widehat{\boldsymbol{\sigma}}_{a}(t) .
$$

The isotropic stress in the above relation is given by

$$
\widehat{\boldsymbol{\sigma}}_{i}(t)=\frac{\widehat{G}}{\widehat{J}}\left(\widehat{\widehat{\boldsymbol{B}}}(t)-\frac{1}{3} \operatorname{tr}(\widehat{\widehat{\boldsymbol{B}}}(t)) \boldsymbol{\delta}\right)+\widehat{K}(\widehat{J}-1) \boldsymbol{\delta}
$$

where $\widehat{J}=\operatorname{det} \widehat{\boldsymbol{F}}(t), \widehat{\widehat{\boldsymbol{B}}}(t)=\widehat{J}^{-2 / 3} \widehat{\boldsymbol{B}}(t)$, with $\widehat{\boldsymbol{B}}(t)=\widehat{\boldsymbol{F}}(t) \widehat{\boldsymbol{F}}^{T}(t)$, while

$$
\widehat{G}=c G^{(1)}+(1-c) G^{(2)}, \quad \widehat{K}=c K^{(1)}+(1-c) K^{(2)},
$$

are respectively the effective shear and bulk moduli of the composite. Note that, for incompressible phases $\left(K^{(1)}, K^{(2)} \longrightarrow \infty\right)$, we have $\widehat{K} \longrightarrow \infty$ and $\widehat{J}=1$, which in turn render the last term in (24) indeterminate, as expected. Note also that, just like the corresponding constitutive equations for the phases, the macroscopic constitutive relation (24) is of the Neo-Hookean form. Furthermore, we remark that expression (24) may be easily extended to constituents more general than Neo-Hookean. The anisotropic stress in (23) is given by

$$
\widehat{\boldsymbol{\sigma}}_{a}(t)=\widehat{\sigma}(t) \widehat{\mathbf{m}}(t) \widehat{\mathbf{m}}(t), \quad \widehat{\mathbf{m}}(t)=\frac{1}{\left|\widehat{\boldsymbol{F}}(t) \cdot \mathbf{m}_{0}\right|} \widehat{\boldsymbol{F}}(t) \cdot \mathbf{m}_{0}, \quad \widehat{\sigma}(t)=\widehat{\sigma}_{0}(t)\left(1+\widehat{\varepsilon}_{0}^{m}(t)\right),
$$

where we recall that $\mathbf{m}_{0}$ is the unit vector along the symmetry axis of transverse isotropy in the undeformed configuration, $\widehat{\varepsilon}_{0}^{m}(t)=\widehat{\lambda}_{m}(t)-1$, with $\widehat{\lambda}_{m}(t)$ denoting the stretch ratio induced by $\widehat{\boldsymbol{F}}(t)$ along the direction $\mathbf{m}_{0}$, and

$$
\widehat{\sigma}_{0}(t)=c \sigma_{0}^{(1)}\left(\widehat{\varepsilon}_{0}^{m}(t), \dot{\hat{\varepsilon}}_{0}^{m}(t)\right)+(1-c) \sigma_{0}^{(2)}\left(\widehat{\varepsilon}_{0}^{m}(t)\right)
$$

is the effective reference axial stress of the composite. Note that, in the above expression (27) the phase response functions $\sigma_{0}^{(r)}$, with $r=1,2$, defined in the previous subsection, must be evaluated at $\widehat{\varepsilon}_{0}^{m}(t)$ and $\dot{\widehat{\varepsilon}}_{0}^{m}(t)$.

At this point, it should be remarked that the Voigt estimate (22) or (23) determines the macroscopic response of a skeletal muscle for completely general 3D loading conditions. This estimate has the advantage of being especially simple and easy to compute, but it also has the disadvantage of being independent of microstructural information other than the volume fractions of the constituent phases. In general, the 
predictions of the Voigt model are known to be stiff and progressively more inaccurate with increasing heterogeneity contrast (i.e., increasingly different phase properties). However, for the special class of incompressible, transversely isotropic materials, the Voigt estimate is known (see, e.g., Agoras et al., 2009) to be exact for axisymmetric loading conditions aligned with the symmetry axis of transverse isotropy, which in the case of the skeletal muscle coincides with the direction of the fibers. In addition, given that muscle response is practically much stiffer in the fiber direction than in the other material directions, for more general loading conditions involving fiber stretching $\left(\widehat{\varepsilon}_{0}^{m}(t) \neq 0\right)$, the axisymmetric mode is expected to have a major effect on the macroscopic behavior of the composite material. Therefore, for general loading conditions with $\widehat{\varepsilon}_{0}^{m}(t) \neq 0$, the Voigt estimate (22) or (23) is also expected to deliver reasonably accurate predictions. This assertion is further investigated in section 4, where the predictions of the model are compared with corresponding full-field FE simulations. On the other hand, for loading conditions in which $\widehat{\varepsilon}_{0}^{m}(t)=0$, i.e., for longitudinal and transverse shear loadings, the estimate (22) or (23) is expected to be accurate for relatively small heterogeneity contrast and to become increasingly more inaccurate with increasing contrast. The development of more accurate estimates for longitudinal and transverse shear loadings would require the use of more sophisticated homogenization techniques (e.g., deBotton et al., 2006; Agoras et al., 2009; Lopez-Pamies and Idiart, 2010; López Jímenez, 2014), accounting properly for microstructural information beyond the volume fractions of the phases (including the shape and distribution of fibers in the transverse plane), and will be pursued in future work.

The constitutive model developed in this section has been implemented in the ABAQUS general-purpose finite element program (ABAQUS, 2009). ABAQUS provides a general interface so that a particular constitutive model can be introduced as a "user subroutine" (UMAT). A detailed description of the model's numerical implementation is provided in the Appendix.

\section{The numerical model}

In this section we present a numerical homogenization approach using a sufficiently complex three dimensional periodic unit cell. Inspired by skeletal muscle histological cross-sections (see for e.g. Sharafi and Blemker (2011)) a muscle structure may be numerically approximated to be composed of identical hexagonal unitcells. The purpose of this numerical analysis is mainly to validate the analytical estimates presented in the previous section. In the present study one of the most critical constraints in the construction of the unit cell is the extremely high volume fraction (usually $>60 \%$ and up to $95 \%$ ) of the muscle fibers together with the finite strains applied in the composite system.

Previous studies have attempted to model the complex distribution and cross-sectional shape of the muscle fibers using anatomical (Sharafi and Blemker, 2010, 2011) or geometrically random structural cells (Virgilio et al., 2015). In spite of those studies we propose, instead, a unit cell (see Fig. 1) that has the 


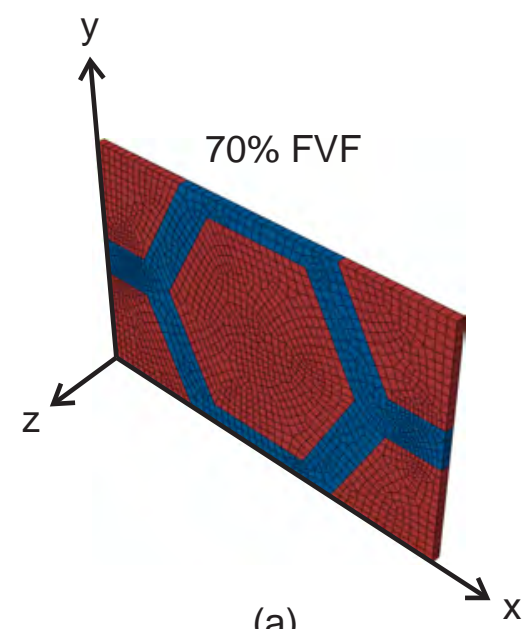

(a)

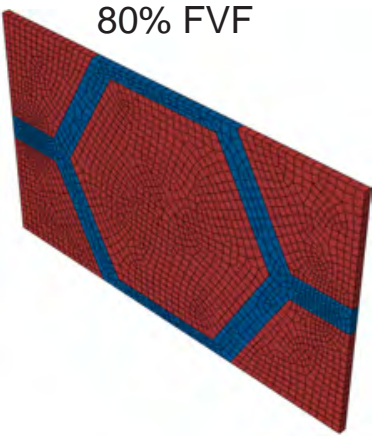

(b)

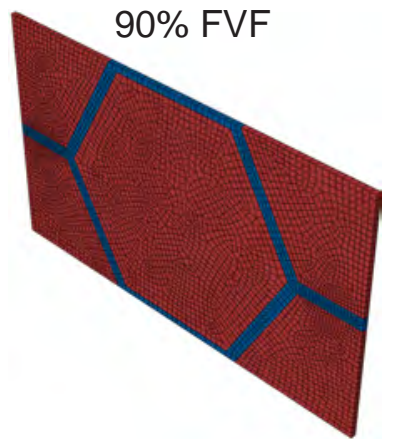

(c)

Figure 1: Periodic hexagonal unit-cell FE meshes with (a) 70\% FVF, (b) $80 \%$ FVF, and (c) $90 \%$ FVF. Fibers and ECM are shown in red and blue colour respectively. Fiber direction is taken along the global z-axis.

following properties:

- the unit cell is periodic, i.e, it is representative and ergodic in the sense of the strict mathematical terminology of homogenization and describes completely the homogenized material response by using appropriate periodic boundary conditions (Michel et al., 1999).

- the cross section of the fibers is hexagonal while the fibers themselves are distributed in a hexagonal pattern allowing to easily reach extremely high volume fractions up to $100 \%$.

- the unit cell is simple enough comprising two complete fibers (one plus four quarters) thus allowing for a good meshing strategy and in general very good mesh quality, which is necessary for the large strain calculations carried out in this study.

- taking into account the invariance of the composite along the fiber direction, only one element is used along this direction, thus reducing the cost of the numerical simulation, while allowing for any three dimensional loading condition that produces homogeneous stress and strain fields along the fiber direction of the tissue.

More specifically, Fig. 1 shows the FE meshes of the unit-cell for fiber volume fraction FVF $=(70 \%$, $80 \%, 90 \%)$ used in the calculations. The constitutive models described in Section 2.1 are used to describe the behavior of the constituents. The fiber size (i.e. superscribed diameter) was taken as $50 \mu \mathrm{m}$ (Sharafi and Blemker, 2011). Eight-node hexahedral hybrid elements (C3D8H in ABAQUS) were utilized in order to handle exactly (in a numerical sense) the incompressible behavior of the muscle fibers and ECM. Also, it was assumed that bonding between fibers and ECM is perfect so that a rigid mesh connection 
between the constituents was applied. A preliminary analysis showed that the behavior of the unit-cell was insensitive to the number of elements used through the out-of-plane direction (fiber direction), thus one element thick meshes were used in the current analyses. Mesh sensitivity studies revealed that meshes with approximately 1,700, 2,200, 3,700 elements for a unit-cell with $70 \%, 80 \%, 90 \%$ FVF respectively (such as the meshes shown in Fig. 1) produce accurate results.

\subsection{Periodic boundary conditions}

In this section we discuss the application of periodic boundary conditions necessary for the analysis of the above described periodic unit cells. These conditions are necessary in order to be able to extract a homogenized response out of the numerical unit cell. In addition, the periodicity conditions directly imply that the proposed unit cell can be infinitely repeated in the three directions to provide a representative muscle structure, whereby requiring only the solution of a single unit cell to estimate the homogenized response.

Practically, this requires the mesh to be identical in all opposite faces of the unit-cell, i.e., the nodes in opposing faces of the unit cell to be one-to-one connected. Consider a three dimensional cuboid unit cell with length axes $\left(L_{1}, L_{2}, L_{3}\right)$. Then, the periodic boundary conditions read (Lopez-Pamies et al., 2013)

$$
\mathbf{u}=(\widehat{\boldsymbol{F}}-\boldsymbol{\delta}) \cdot \mathbf{X}+\mathbf{u}^{*}
$$

where the second-order tensor $\widehat{\boldsymbol{F}}$ denotes the average deformation gradient of the unit-cell which is equal to the applied deformation gradient, $\mathbf{X}$ denotes the spatial coordinates in the reference configuration, and $\mathbf{u}^{*}$ is a periodic displacement field. ${ }^{1}$

Next, one needs to fix one node to cancel the rigid body motion in the FE calculations. For convenience, we choose this node to be at the origin such that $u_{i}(0,0,0)=0(i=1,3)$.

Subsequently, one can subtract the nodal displacements of opposite boundary sides so that we get the following nodal constraints for the corner nodes of the unit-cell $\forall i=1,3$ (Mbiakop et al., 2015)

$$
\begin{aligned}
& u_{i}\left(L_{1}, 0,0\right)-u_{i}(0,0,0)=\left(\widehat{F}_{i 1}-\delta_{i 1}\right) L_{1} \\
& u_{i}\left(0, L_{2}, 0\right)-u_{i}(0,0,0)=\left(\widehat{F}_{i 2}-\delta_{i 2}\right) L_{2} \\
& u_{i}\left(0,0, L_{3}\right)-u_{i}(0,0,0)=\left(\widehat{F}_{i 3}-\delta_{i 3}\right) L_{3}
\end{aligned}
$$

The above simple relations show that the displacement components of the corner nodes $\left(L_{1}, 0,0\right),\left(0, L_{2}, 0\right)$ and $\left(0,0, L_{3}\right)$ are one-to-one connected to the average deformation gradient $\widehat{\boldsymbol{F}}$. Then, one can write the constraint equations for the rest of the nodes lying on opposite faces of the unit-cell making use of Eq. (29),

\footnotetext{
${ }^{1}$ For a detailed proof of the fact that the applied $\widehat{\boldsymbol{F}}$ corresponds to the volume average of $\boldsymbol{F}$ in the unit cell the reader is referred to Michel et al. (1999) and Javili et al. (2013).
} 
i.e., $\forall i=1,3$

$$
\begin{aligned}
& u_{i}\left(L_{1}, x_{2}, x_{3}\right)-u_{i}\left(0, x_{2}, x_{3}\right)=\left(\widehat{F}_{i 1}-\delta_{i 1}\right) L_{1}=u_{i}\left(L_{1}, 0,0\right) \\
& u_{i}\left(x_{1}, L_{2}, x_{3}\right)-u_{i}\left(x_{1}, 0, x_{3}\right)=\left(\widehat{F}_{i 2}-\delta_{i 2}\right) L_{2}=u_{i}\left(0, L_{2}, 0\right) \\
& u_{i}\left(x_{1}, x_{2}, L_{3}\right)-u_{i}\left(x_{1}, x_{2}, 0\right)=\left(\widehat{F}_{i 3}-\delta_{i 3}\right) L_{3}=u_{i}\left(0,0, L_{3}\right)
\end{aligned}
$$

The above algebraic analysis reveals that all periodic linear constraints between all nodes can be written in terms of the displacements of three corner nodes, i.e., $u_{i}\left(L_{1}, 0,0\right), u_{i}\left(0, L_{2}, 0\right)$ and $u_{i}\left(0,0, L_{3}\right)$, which, in turn, are given in terms of $\widehat{\boldsymbol{F}}$ by Eq. (29). This, further, implies that the only nodes that boundary conditions need to be applied to are lying on opposite faces of the unit-cell $\left(L_{1}, 0,0\right),\left(0, L_{2}, 0\right)$ and $\left(0,0, L_{3}\right)$ (together with the axes origin $(0,0,0)$ which is fixed). In ABAQUS the periodicity constraints are enforced through the "EQUATION" option.

The volume average true stress tensor in the unit-cell $\widehat{\boldsymbol{\sigma}}$ is calculated as $\widehat{\boldsymbol{\sigma}}=\sum_{i} \boldsymbol{\sigma}_{i} V_{i} / \sum_{i} V_{i}$ where $\sigma_{i}$ and $V_{i}$ are the true stress tensor evaluated at the centroid and the volume of the $i$-th finite element respectively. The volume average nominal (1st Piola-Kirchhoff) stress tensor $\widehat{t}$ is then computed by the relation $\widehat{\boldsymbol{t}}=\widehat{J} \widehat{\boldsymbol{F}}^{-1} \cdot \widehat{\boldsymbol{\sigma}}$. Here, $\widehat{J}=\operatorname{det} \widehat{\boldsymbol{F}}$, where $\widehat{\boldsymbol{F}}$ corresponds to the average deformation gradient in the unit cell and has been defined via the periodic boundary conditions in Eq. (28) and is directly linked to the displacement of the corner nodes $\left(L_{1}, 0,0\right),\left(0, L_{2}, 0\right)$ and $\left(0,0, L_{3}\right)$, as discussed in the context of Eq. (29). All loadings are characterized by the macroscopic applied average deformation gradient $\widehat{\boldsymbol{F}}$.

\section{Results}

In this section, we compare the predictions of the analytical model for the skeletal muscle developed in section 2 with corresponding experimental results available from the literature and FE calculations based on the unit-cell analysis discussed in section 3. In addition, we examine the effect of muscle fiber's elastic modulus, ECM's stiffness in its preferred direction, declination angle between the muscle fibers direction and the ECM's preferred direction, and the velocity of contraction on the effective muscle response. The model parameters used in the calculations of this section are given in the following subsection.

\subsection{Model parameters}

In the current study we assume that muscle response under passive compression is associated with the isotropic (Neo-Hookean) behavior of muscle fibers and ECM. The macroscopic shear modulus of the muscle $\widehat{G}$ can be determined from uniaxial compression experiments on whole muscle tissues, such as those reported in Van Loocke et al. (2006). However, experimental measurements for the shear moduli of the constituents themselves do not currently exist in the literature. A previous work only suggests that the shear modulus of endomysium is of the same order of magnitude as that of muscle (Sharafi and Blemker, 2011). Therefore, we examine skeletal muscle response over a range of shear moduli contrasts between 
muscle fiber and $\operatorname{ECM} G^{(1)} / G^{(2)}=2,5,10$, with $G^{(2)}=1 \mathrm{kPa}$. Also, we assign the corresponding bulk modulus to be 10,000 times the shear modulus in order to account for the incompressible behavior of the constituents $\left(K^{(r)}=10^{4} G^{(r)}, r=1,2\right)$.

For the anisotropic part of muscle fibers we need to set the values for $\sigma_{\max }$ and the parameters that enter the functions $f_{e}, f_{r}$, and $f_{p}^{(1)}$ in Eqs. (11)-(13). Here, the values for $\sigma_{\max }$, as well as $\varepsilon_{0}^{m, o p t}$ and $\varepsilon_{0}^{m, m i n}$ that enter the active stress-strain function $f_{e}$ are directly obtained from the experimental measurements of Hawkins and Bey (1994) on rat tibialis anterior muscles. These experimental results are further used for comparison with the analytical model predictions in Section 4.2. The values for the parameters $\dot{\varepsilon}_{0}^{m, \text { min }}, k_{c}, k_{e}, d$ that enter the active stress-strain rate function $f_{r}$ are taken from Böl and Reese (2008). The muscle fiber's passive elastic modulus $E_{p}$ that enters the passive stress-strain function $f_{p}^{(1)}$ is obtained from the single fiber experimental results of Thacker et al. (2012) on rat tibialis anterior muscles. Also, we assume fully active muscle fibers $\left(f_{a}=1\right)$ when the activity of the muscle is taken into account in the analysis and $f_{a}=0$ when muscle exhibits only passive behavior.

Table 1: Model parameters used in the analysis

\begin{tabular}{ccc}
\multicolumn{2}{c}{ Table 1: Model parameters used in the analysis } \\
\hline \hline Parameter & Value & Reference \\
\hline$\widehat{G}$ & $2.5 \mathrm{kPa}$ & Van Loocke et al. (2006) \\
$\sigma_{\max }$ & $73 \mathrm{kPa}$ & Hawkins and Bey (1994) \\
$\varepsilon_{0}^{m, m i n}$ & -0.318 & Hawkins and Bey (1994) \\
$\varepsilon_{0}^{m, o p t}$ & 0.192 & Hawkins and Bey (1994) \\
$\dot{\varepsilon}_{0}^{m, \min }$ & $-17 \mathrm{~s}^{-1}$ & Böl and Reese (2008) \\
$k_{c}$ & 5 & Böl and Reese (2008) \\
$k_{e}$ & 5 & Böl and Reese (2008) \\
$d$ & 1.5 & Böl and Reese (2008) \\
$E_{p}$ & $63 \mathrm{kPa}$ & Thacker et al. (2012) \\
$A 1$ & $53 \mathrm{kPa}$ & Holzapfel et al. (2005) \\
$A 2$ & 110 & Holzapfel et al. (2005) \\
$\theta_{0}$ & $59^{\circ}$ & Purslow and Trotter (1994) \\
$c$ & 0.95 & Lieber et al. (2003) \\
\hline & &
\end{tabular}

Regarding skeletal muscle's ECM anisotropic behavior we need to assign the values for the parameters $A 1, A 2$ and the angle $\theta_{0}$ that enter the function $f_{p}^{(2)}$ in Eq. (16). Here we follow Bleiler et al. (2015) where the parameters $A 1, A 2$ are fitted to uniaxial extension measurements on collagenous tissues reported in Holzapfel et al. (2005). In particular, we adopt the data of Holzapfel et al. (2005) for the ventro-lateral 


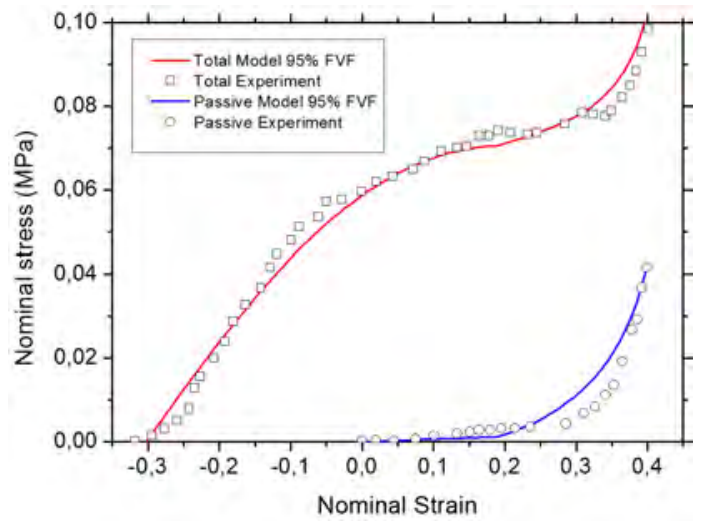

Figure 2: Muscle response under loadings in the muscle fiber direction. Analytical model predictions in comparison with the experimental data of Hawkins and Bey (1994).

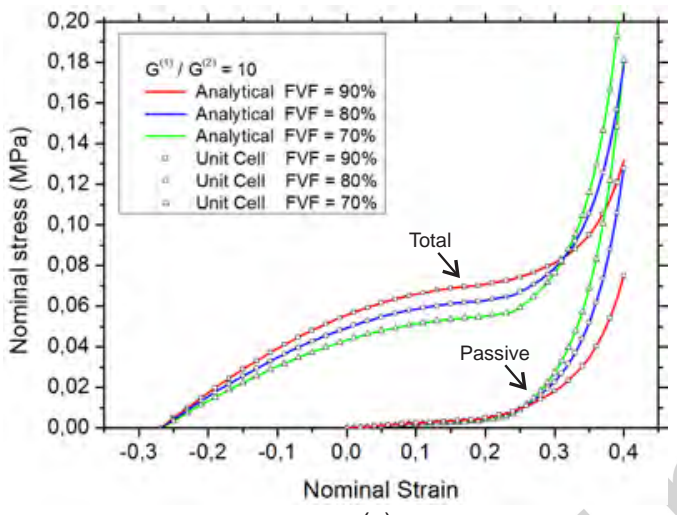

(a)

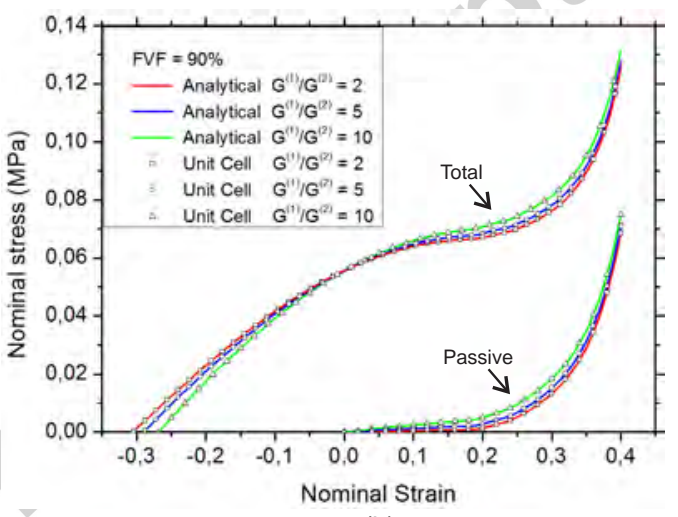

(b)

Figure 3: Muscle response under loadings in the muscle fiber direction. Analytical model predictions compared to FE unit-cell simulations for (a) $G^{(1)} / G^{(2)}=10$ and various $\mathrm{FVF}$, and (b) FVF $=90 \%$ and various shear moduli contrasts $G^{(1)} / G^{(2)}$. The shear modulus of ECM is taken as $G^{(2)}=1 \mathrm{kPa}$.

external region of human lumbar anulus fibrosus which maximize the agreement between the model and the experimental results of Hawkins and Bey (1994). Also, we account for an angle $\theta_{0}$ between the muscle fiber direction and the ECM's fiber network preferred direction based on Purslow and Trotter (1994).

Finally, the value for the fiber volume fraction $c$ that appears in Eq. (22) is chosen according to Lieber et al. (2003) for healthy muscles. All the input data used in the current analysis are summarized in Table 1.

\subsection{Comparison with experimental data and FE simulations}

The analytical homogenization model is used to predict muscle response under uniaxial loading where the muscle fiber direction coincides with the loading direction. The model predictions are compared to the experimental results of Hawkins and Bey (1994), who measured the passive and active stress-strain response 
of a healthy rat tibialis anterior muscle. The experimental data were obtained under isometric conditions, thus the strain rate dependence on the muscle fiber stress is omitted $\left(f_{r}\left(\dot{\varepsilon}_{0}^{m}\right)=1\right)$. All the rest input data used in the model are shown in Table 1 and described in Section 4.1. Fig. 2 shows a very good agreement between the model predictions and the experiments for both total (active and passive) and passive muscle response.

The influence of fiber volume fraction and shear moduli contrast on the effective muscle response in the muscle fiber direction is shown in Fig. 3. Comparison between the analytical model predictions and the FE unit-cell calculations shows excellent agreement (Fig. 3). Fig. 3a shows that increased volume fractions of ECM in a muscle may result in a manifold increase in muscle stress output when muscle is stretched in the fiber direction. A cross-over of the total response occurs at large enough tensile strains; while at smaller strains, the higher FVF leads to higher stresses, this response is reversed at larger strains $(\sim>0.3)$. This cross-over is directly attributed to the increasing role of the ECM part, which becomes rather important at larger strains and tends to lead to higher stresses for lower FVF. On the other hand, the shear moduli contrast between muscle fibers and ECM (and thus the isotropic behavior of the constituents) seems to have only a weak effect on the total anisotropic tissue response, as shown in Fig. 3b.

In addition to loadings in the fiber direction, the analytical model is also able to capture the muscle response under shear loadings across the fibers (perpendicular shear) (Fig. 4), as shown in Fig. 5. In particular, we examine the material responses under the shear deformations $\widehat{F}_{x z}$ and $\widehat{F}_{y z}$, where z-axis is aligned in the undeformed fiber direction, for various fiber volume fractions and shear moduli contrasts between the constituents. It is noted that analytical and FE unit-cell calculations result in the same muscle response under either $\widehat{F}_{x z}$ or $\widehat{F}_{y z}$ loading. The comparisons between the analytical and numerical model predictions presented in Fig. 5 show a very good agreement especially in the case where the activity of the muscle fibers is taken into account. Fig. 5 also indicates that the estimates of the analytical model under shear loadings become less accurate as the FVF is decreased and at the same time the shear moduli contrast is increased. The analysis results show that muscle fibers control the overall muscle response when shear strains are maintained under a certain threshold $(\simeq 0.7-0.8$ in the present case) whereas above this threshold the effective response becomes dominated by ECM's behavior.

Note that perpendicular shear is the only mode of simple shear in which the fibers are stretched, thus causing a stress to develop in the direction normal to the applied shear deformation. Fig. 6 shows that, for various FVF, a positive normal stress in the undeformed muscle fiber direction is developed when the fibers are either active or passive. In addition, this normal stress appears to be in the same order of magnitude with the corresponding shear stress. 


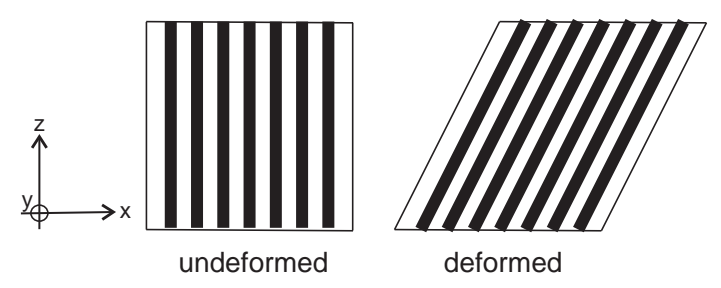

(a)

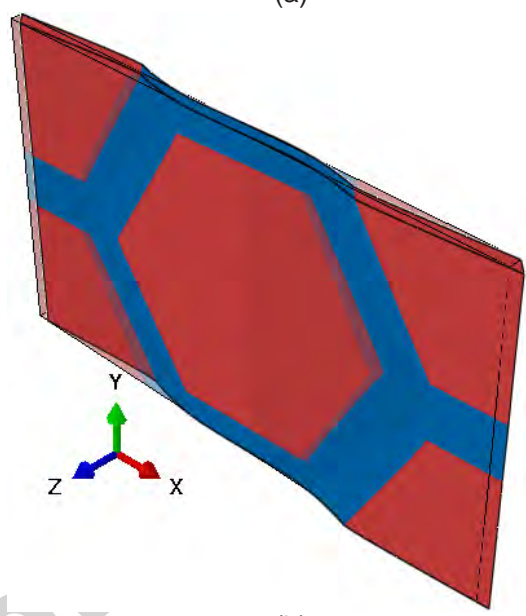

(b)

Figure 4: Schematic representation of perpendicular (out-of-plane) simple shear loading $\widehat{F}_{x z}$ : (a) 2D sketch of undeformed and deformed configurations (the dark lines represent the fibers) and (b) superimposed undeformed and deformed shape of the unit-cell with $70 \% \mathrm{FVF}$ and $G^{(1)} / G^{(2)}=2$. Fibers and ECM are shown in red and blue colour respectively. 

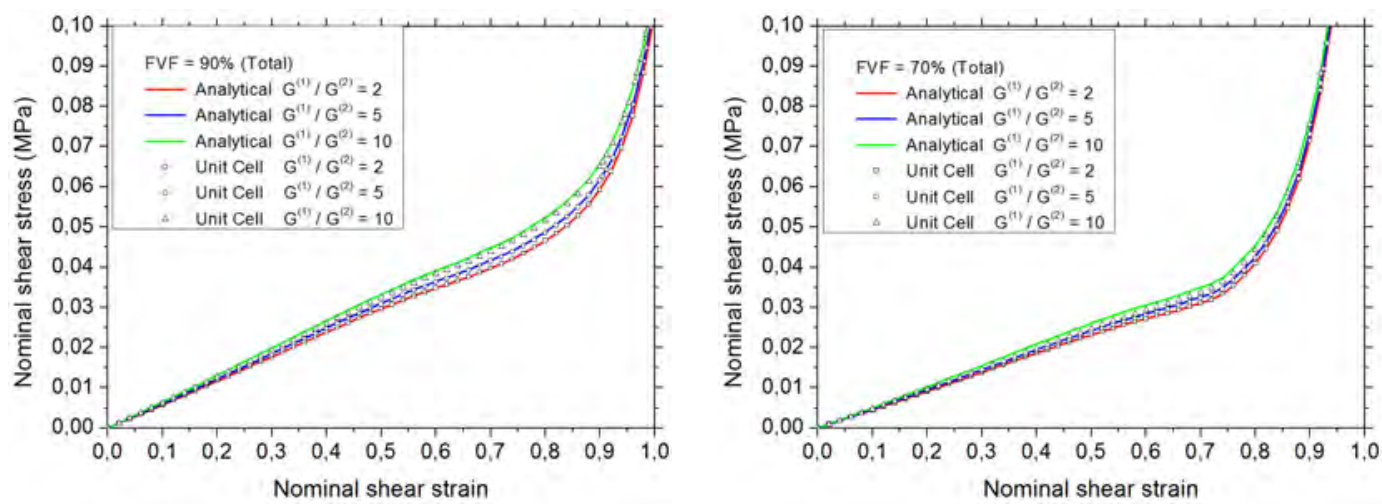

(a)
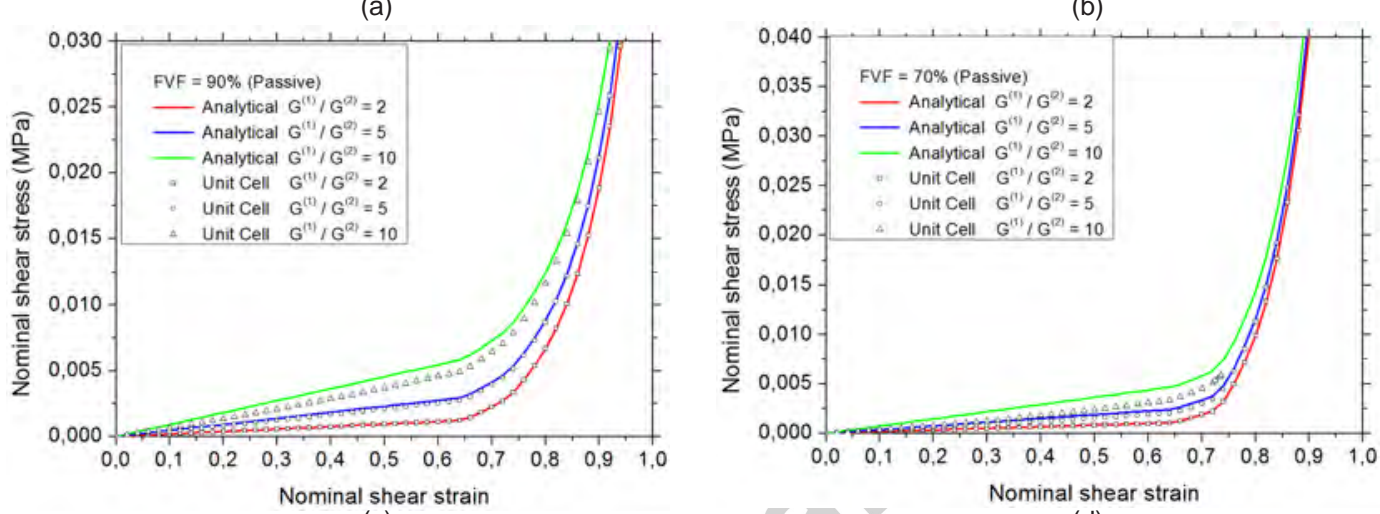

(c)

(d)

Figure 5: Perpendicular simple shear total (active and passive) and passive behavior under either $\widehat{F}_{x z}$ or $\widehat{F}_{y z}$ loading for various shear moduli contrasts when (a and c) $\mathrm{FVF}=90 \%$, and ( $\mathrm{b}$ and $\mathrm{d}$ ) $\mathrm{FVF}=70 \%$.

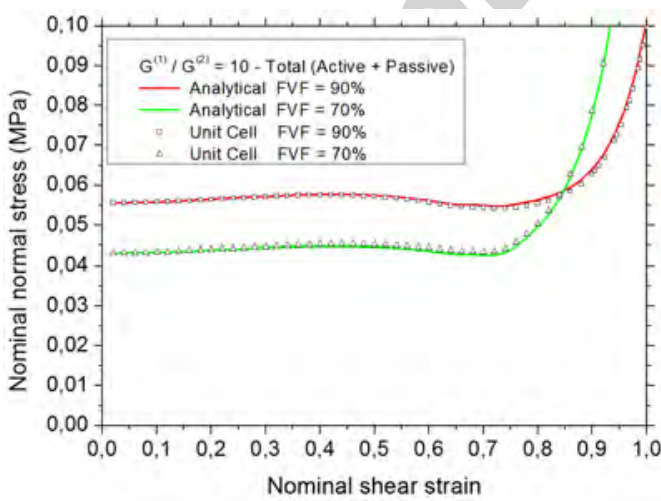

(a)

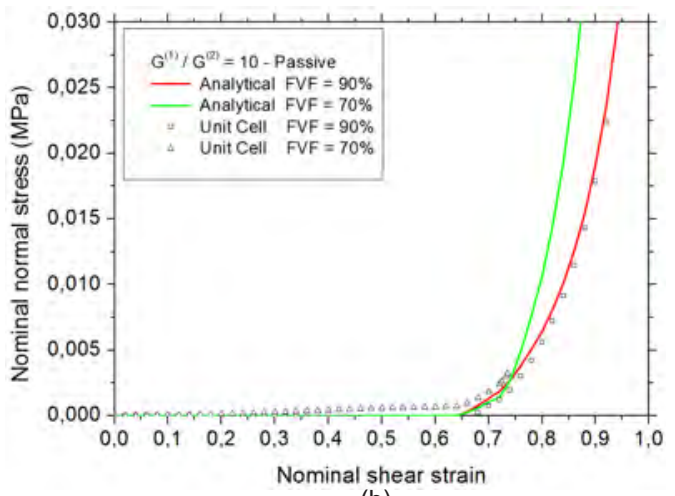

(b)

Figure 6: Normal stress-shear strain behavior under perpendicular simple shear loading $\widehat{F}_{x z}$ or $\widehat{F}_{y z}$ for various FVF when (a) muscle fibers are fully active, and (b) muscle fibers remain passive. In both cases $G^{(1)} / G^{(2)}=10$. 


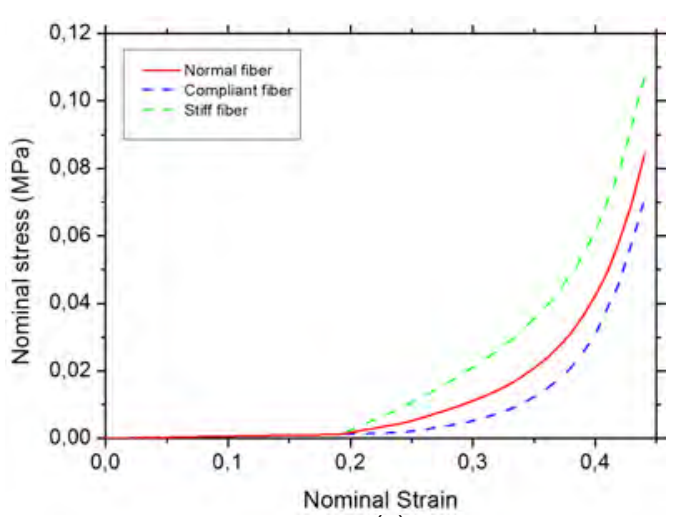

(a)

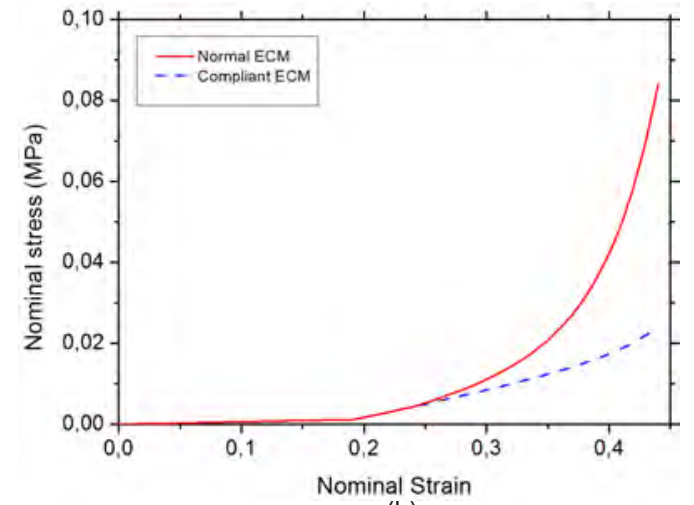

(b)

Figure 7: Anisotropic muscle stress-strain response dependence on (a) muscle fiber's elastic modulus, and (b) ECM's stiffness in its preferred direction. The values $E_{p}=160 \mathrm{kPa}$ (stiff) and $E_{p}=6 \mathrm{kPa}$ (compliant) were chosen for muscle fiber's elastic modulus as the highest and lowest values among various muscles reported in Gillies and Lieber (2011). Based on the experimental data of Holzapfel et al. (2005) a tenfold decrease of the parameter $A 1$ in Eq. (16), i.e. $A 1=5.3 k P a$ was assumed realistic to represent a more compliant behavior in ECM's preferred direction.

\subsection{Influence of material parameters on muscle response}

In the previous section the analytical model for skeletal muscle has been validated through experimental and FE results. In this section we will focus on the analytical model estimates only and explore the effect of a number of parameters including muscle fiber's passive elastic modulus, ECM's stiffness in its preferred direction, declination angle between the muscle fibers direction and the ECM's preferred direction, and the velocity of contraction. The goal is to reveal the influence of micromechanical parameters on the macroscopic muscle behavior. The parameter values presented in Table 1 are used in the analysis and the strain rate dependence on active muscle fiber stress $\left(\dot{\varepsilon}_{0}^{m} \neq 0\right)$ is only considered when the influence of velocity of contraction is examined.

Fig. 7 shows the effects of muscle fiber's passive elastic modulus and ECM's stiffness in its preferred direction on muscle anisotropic behavior. Muscle response seems to be weakly dependent on the muscle's fiber modulus (Fig. 7a). On the other hand, in Fig. 7b we observe a strong dependence of the effective response on ECM's stiffness. An even stronger dependence of the effective response upon the angle $\theta_{0}$ between the muscle fibers direction and the ECM's preferred direction can be observed in Fig. 8a. Fig. 8a shows that even a small change in $\theta_{0}$ may lead to orders of magnitude different muscle stress output when muscle is stretched in the fiber direction. Finally, in Fig. 8b we examine the influence of velocity of contraction on muscle's response. To do this we calculate again muscle stress from Eq. (9) by assuming that muscle shortens or extends with a constant strain rate $\left(\dot{\varepsilon}_{0}^{m} \neq 0\right)$ based on the function $f_{r}\left(\dot{\varepsilon}_{0}^{m}\right)$ (Eq. (12)). The isometric case $\left(\dot{\varepsilon}_{0}^{m}=0\right)$ is also included in the same figure for illustration. The values used in the current analysis for the parameters $\dot{\varepsilon}_{0}^{m, \text { min }}, k_{c}, k_{e}, d$ that enter the function $f_{r}$ are presented in Table 1. 


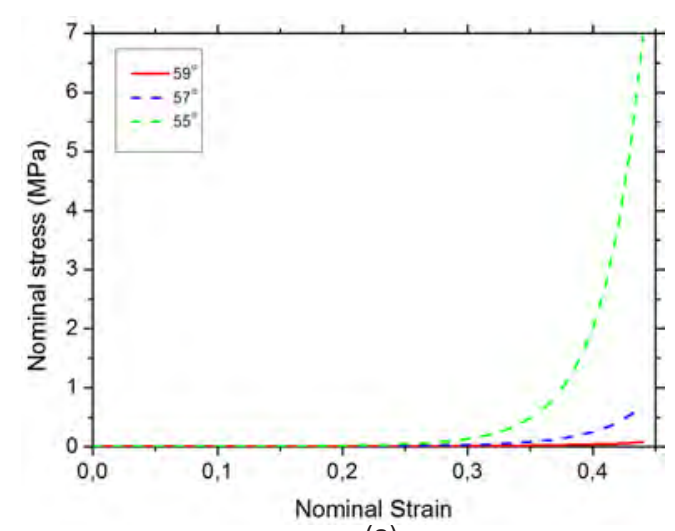

(a)

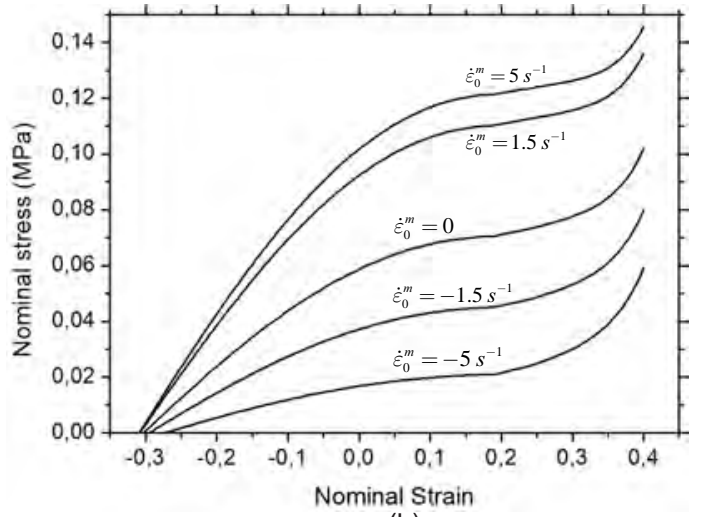

(b)

Figure 8: Anisotropic muscle stress-strain response dependence on (a) the angle $\theta_{0}$ between the muscle fibers direction and the ECM's preferred direction, and (b) velocity of active contraction.

\section{Discussion}

An analytical constitutive model incorporating microstructural characteristics has been developed for skeletal muscles subjected to three-dimensional loading conditions. In order to achieve this goal a straightforward homogenization scheme has been used to bridge the different length scales of the muscle structure. Due to its simplicity and numerical efficiency, the proposed muscle model has been numerically implemented in a user-material subroutine which, in turn, allows for the simulation of three dimensional geometries of any complexity, which is critical for biological systems.

The muscle model presented in this study has been validated by comparison with experimental data and full field FE calculations of periodic unit-cells. The model has been found to be in very good agreement with the experimental results and the FE results for a range of fiber volume fractions and loading conditions. The analysis revealed that increased fractions of ECM in a muscle volume may lead to a significant stiffer muscle response. This finding is in accord with Smith et al. (2011) who showed that ECM's passive behavior has significant implications in the increased muscle stiffness of spastic muscles in patients with cerebral palsy. Since FVF depends on whether the muscle is healthy or diseased (i.e. decreasing FVF with diseased muscles (Lieber et al., 2003)), it is important that the analytical model can predict muscle behavior accurately within a vast range of fiber volume fractions and different material properties of the constituents.

In line with the experimental study of Meyer and Lieber (2011) the model has been able to predict that ECM's stiff and highly nonlinear behavior is mainly responsible for the total muscle response under stretching in the fiber direction. Also, the model has been able to shed light on how muscle's macroscopic response is affected by changes of the muscle fibers' elastic modulus, ECM's stiffness in its preferred direction, and declination angle between the muscle fibers direction and the ECM's preferred direction. An important finding of this work is that the muscle response when muscle fibers become stretched is significantly more 
influenced by ECM's material properties (stiffness and preferred orientation with respect to muscle fibers) than the muscle's fibers modulus, even if ECM accounts for only $5 \%$ of the muscle volume. A characteristic cross-over is taking place between curves of different volume fractions at large strains where the ECM response becomes important. In this large strain range, the homogenized response of the fiber-ECM system gives higher stresses with decreasing FVF, while this effect is reversed at lower strains where the FVF controls the response. Furthermore, it has been shown that significant differences in muscle response arise between isometric and non-isometric contractions as a result of the strain rate dependence on the active muscle fiber stress.

Most materials exhibit the so-called positive Poynting effect in the sense that under simple shear loading conditions the material tends to expand in the direction normal to the applied shear deformation. However, in fiber-reinforced soft tissues under perpendicular shear loading, recent experimental (Janmey et al., 2007) and theoretical (Destrade et al., 2014) findings suggest the presence of a significant negative or reverse Poynting effect which causes the sheared faces of the material to tend to come together; in that case a tensile normal stress in the direction perpendicular to the shear stress is developed as a consequence of the simple shear deformation mode. In this study, the model has been able to predict the significant reverse Poynting effect under perpendicular simple shear loading which is accompanied with the development of positive (tensile) normal stress in the undeformed fiber direction. As in previous studies, the normal and shear stresses have been found to be of the same order of magnitude.

In view of the presented analytical model, we remark that equation (27) in Section 2.2 can be easily modified to compute muscle force instead of muscle stress in the fiber direction. The 1-D equation for muscle force, which then includes explicitly the volume fraction of fibers in the ECM, can be incorporated into rigid-body musculoskeletal modeling frameworks through modified Hill-type muscle models in order to be able to study how the volume fractions and material properties of muscle constituents would affect the whole body movement.

ECM's behavior was simplified to be transversely isotropic assuming a single preferred direction in the material. For a more realistic material description this model can be substituted by any other model that takes into account the internal microstructure of ECM, i.e., the presence of a fiber network at a much smaller length scale than that of the muscle fiber itself (e.g. Chen et al., 2011; deBotton and Oren, 2013; Tkachuk and Linder, 2012). Also, due to lack of direct measurements on muscle's connective tissue behavior (especially those for endomysium and perimysium which mostly interact with muscle fibers), experimental data for another collagenous tissue (human lumbar anulus fibrosus) were used to describe ECM's behavior in this study. Nonetheless, the data adopted here reflect the high nonlinear and much stiffer than the muscle fibers passive behavior of muscle's ECM as indirectly determined by Meyer and Lieber (2011).

In the present work, a Voigt homogenization scheme has been employed to bridge the different scales of the muscle tissue. This scheme is known to account for the microstructure only through the volume fraction of 
the phases. For loading conditions involving no fiber stretching, i.e., longitudinal and in-plane shear loadings, the proposed Voigt-type model is expected to deliver predictions that are stiff and become progressively more inaccurate with increasing heterogeneity contrast. In order to overcome this shortcoming, the development of more sophisticated homogenization schemes accounting for microstructural information beyond the volume fractions of the phases is required. Recent progress in modeling fiber-reinforced elastomers (e.g. Agoras et al., 2009; deBotton et al., 2006; López Jímenez, 2014; Lopez-Pamies and Idiart, 2010) may prove useful to this end. This possibility will be investigated in future work.

It should be remarked that both the numerical and the analytical models proposed in this work involve the assumption that the muscle-fiber and ECM phases are initially perfectly bonded and remain perfectly bonded throughout any given loading. This hypothesis is expected to be reasonably accurate, in general (i.e., for situations in which the muscle tissue is healthy and subjected to normal loading conditions). However, in situations where the applied loads lead to excessively large tissue stresses or strains (e.g. during active lengthening contraction) or when muscle tissue suffers from a disease (e.g. Duchenne muscular dystrophy), local interfacial debonding may occur due to local damage of the transmembrane connections of the phases (Gao et al., 2008; Virgilio et al., 2015). In general, the imperfect phase bonding is expected to lead to a softer macroscopic response for the skeletal muscle. It would certainly be of interest to investigate the effect of imperfect phase bonding. Specifically, in the context of a numerical homogenization scheme, the FE unitcells presented in Section 3 can be altered to include phase boundary imperfections by either accounting for a third thin solid interphase at the fiber-ECM boundaries or by connecting the coincident boundary mesh nodes of fiber and ECM with springs of different stiffnesses (Spring and Paulino, 2015; Virgilio et al., 2015; Würkner et al., 2013). These aspects will be examined in a future work. It should be emphasized, however, that accounting for imperfect bonding in the context of an analytical homogenization model is a non-trivial task (see, e.g., related work by Bigoni et al., 1998; Hashin, 1990, 1992, for linear elastic composites).

Finally, it should be pointed out that several important muscle features, such as different fiber types (fastor slow-twitch), grouping of fibers in motor units, fiber recruitment, muscle fatigue, and muscle residual force enhancement are not included in the present model. The addition of such features in the model is beyond the scope of this study and will be taken into account in a future work.

\section{Conclusions}

A new constitutive model for skeletal muscle tissue taking into account the volume fractions and the material properties of its constituents is developed. Despite the limitations of the present model, to the best knowledge of the authors, this is the first microstructural continuum model in the literature which is able to describe the macroscopic active and passive behavior of skeletal muscles. The model is used to shed light on how several micromechanical changes may affect muscle's macroscopic behavior. Our analysis, in accord 
with former literature, suggests that changes in intramuscular connective tissue may have a significant role in the increased muscle stiffness resulted by diseases, such as spasticity. Such observations may lead to new therapeutic treatments on skeletal muscle disease-related dysfunctions. In addition, the constitutive model is numerically implemented in a finite-element framework which provides the opportunity to investigate clinical related problems in a multiscale framework by analyzing three-dimensional anatomical muscle structures.

\section{Acknowledgments}

K.D. would like to acknowledge partial support from the European Research Council (ERC) under the European Unions Horizon 2020 research and innovation program (Grant Agreement No. 636903).

\section{Appendix}

In this appendix we describe the numerical implementation of the analytical constitutive model in the context of the finite element method. In view of the non-linearity and the rate dependence of the model, the finite element solution is developed incrementally. The constitutive calculations are carried out at the element Gauss integration points. In particular, at a given Gauss point, the directional vector in the reference configuration $\mathbf{m}_{0}$, the homogenized solution $\left(\widehat{\boldsymbol{F}}_{n}, \widehat{\boldsymbol{\sigma}}_{n},\left.\widehat{\varepsilon}_{0}^{m}\right|_{n}\right)$ at time $t_{n}$ as well as the average deformation gradient $\widehat{\boldsymbol{F}}_{n+1}$ at time $t_{n+1}=t_{n}+\Delta t$ are known, and the problem is to determine $\left(\widehat{\boldsymbol{\sigma}}_{n+1},\left.\widehat{\varepsilon}_{0}^{m}\right|_{n+1}, \widehat{\mathbf{m}}_{n+1}\right)$.

The constitutive calculations at the Gauss points are carried out in the following order:

$$
\begin{aligned}
& \widehat{\boldsymbol{F}}_{n+1}=\left(\operatorname{det} \widehat{\boldsymbol{F}}_{n+1}\right)^{-1 / 3} \widehat{\boldsymbol{F}}_{n+1}, \\
& \widehat{\widehat{\boldsymbol{B}}}_{n+1}=\overline{\widehat{\boldsymbol{F}}}_{n+1} \cdot \overline{\widehat{\boldsymbol{F}}}_{n+1}^{T}, \widehat{\boldsymbol{F}}_{n+1} \cdot \mathbf{m}_{0}, \\
& \widehat{\mathbf{m}}_{n+1}=\frac{1}{\left|\widehat{\boldsymbol{F}}_{n+1} \cdot \mathbf{m}_{0}\right|} \\
& \left.\widehat{\lambda}_{m}\right|_{n+1}=\sqrt{\mathbf{m}_{0} \cdot \widehat{\boldsymbol{F}}_{n+1}^{T} \cdot \widehat{\boldsymbol{F}}_{n+1} \cdot \mathbf{m}_{0}}, \\
& \left.\widehat{\varepsilon}_{0}^{m}\right|_{n+1}=\left.\widehat{\lambda}_{m}\right|_{n+1}-1, \\
& \left.\dot{\hat{\varepsilon}}_{0}^{m}\right|_{n+1}=\frac{\left.\widehat{\varepsilon}_{0}^{m}\right|_{n+1}-\left.\widehat{\varepsilon}_{0}^{m}\right|_{n}}{\Delta t}, \\
& \widehat{G}=c \cdot G^{(1)}+(1-c) \cdot G^{(2)}, \\
& \widehat{K}=c \cdot K^{(1)}+(1-c) \cdot K^{(2)}, \\
& \left.\sigma_{0}^{(1)}\right|_{n+1}=\left.\sigma_{\max } f_{a}\right|_{n+1} f_{e}\left(\left.\widehat{\varepsilon}_{0}^{m}\right|_{n+1}\right) f_{r}\left(\left.\dot{\varepsilon}_{0}^{m}\right|_{n+1}\right)+f_{p}^{(1)}\left(\left.\widehat{\varepsilon}_{0}^{m}\right|_{n+1}\right),
\end{aligned}
$$




$$
\begin{aligned}
& \left.\sigma_{0}^{(2)}\right|_{n+1}=f_{p}^{(2)}\left(\left.\widehat{\lambda}_{m}\right|_{n+1}, \theta_{0}\right), \\
& \left.\widehat{\sigma}_{0}\right|_{n+1}=\left.c \cdot \sigma_{0}^{(1)}\right|_{n+1}+\left.(1-c) \cdot \sigma_{0}^{(2)}\right|_{n+1}, \\
& \widehat{\sigma}_{n+1}=\left.\left(1+\left.\widehat{\varepsilon}_{0}^{m}\right|_{n+1}\right) \widehat{\sigma}_{0}\right|_{n+1}, \\
& \left.\widehat{\boldsymbol{\sigma}}_{i}\right|_{n+1}=\frac{\widehat{G}}{\operatorname{det} \widehat{\boldsymbol{F}}_{n+1}}\left(\widehat{\widehat{\boldsymbol{B}}}_{n+1}-\frac{1}{3} \operatorname{tr}\left(\widehat{\boldsymbol{B}}_{n+1}\right) \boldsymbol{\delta}\right)+\widehat{K}\left(\operatorname{det} \widehat{\boldsymbol{F}}_{n+1}-1\right) \boldsymbol{\delta}, \\
& \left.\widehat{\boldsymbol{\sigma}}_{a}\right|_{n+1}=\widehat{\sigma}_{n+1} \widehat{\mathbf{m}}_{n+1} \widehat{\mathbf{m}}_{n+1}, \\
& \left.\widehat{\boldsymbol{\sigma}}\right|_{n+1}=\left.\widehat{\boldsymbol{\sigma}}_{i}\right|_{n+1}+\left.\widehat{\boldsymbol{\sigma}}_{a}\right|_{n+1} .
\end{aligned}
$$

The "implicit" version of ABAQUS is used, in which the finite element formulation is based on the weak form of the equilibrium equations, the solution is carried out incrementally, and the discretized nonlinear equations are solved by using Newton's method. The Jacobian of the equilibrium Newton-loop requires the so-called "linearization moduli" of the algorithm that handles the constitutive equations; these moduli are defined in terms of a fourth-order tensor $\widehat{\mathcal{C}}$ that relates the variation of stress $\partial \widehat{\boldsymbol{\sigma}}$ to the variation of strain $\partial \widehat{\varepsilon}$ over the increment under consideration:

$$
\partial \widehat{\boldsymbol{\sigma}}=\widehat{\mathcal{C}}: \partial \widehat{\boldsymbol{\varepsilon}}=\left(\widehat{\mathcal{C}}^{i}+\widehat{\mathcal{C}}^{a}\right): \partial \widehat{\mathcal{\varepsilon}}
$$

The details of the derivation of $\widehat{\mathcal{C}}^{i}$ and $\widehat{\mathcal{C}}^{a}$ are reported in Suchocki (2011) and Spyrou (2009) respectively. Here we note that

$\widehat{\boldsymbol{\mathcal { C }}}_{i j k l}^{i}=\frac{\widehat{G}}{\widehat{J}}\left[\frac{1}{2}\left(\delta_{i k} \overline{\boldsymbol{B}}_{j l}+\overline{\widehat{\boldsymbol{B}}}_{i k} \delta_{j l}+\delta_{i l} \overline{\boldsymbol{B}}_{j k}+\widehat{\widehat{\boldsymbol{B}}}_{i l} \delta_{j k}\right)-\frac{2}{3} \delta_{i j} \overline{\boldsymbol{B}}_{k l}-\frac{2}{3} \overline{\boldsymbol{B}}_{i j} \delta_{k l}+\frac{2}{9} \delta_{i j} \delta_{k l} \overline{\boldsymbol{B}}_{m m}\right]+\widehat{K}(2 \widehat{J}-1) \delta_{i j} \delta_{k l}$,

and

$$
\widehat{\mathcal{C}}_{i j k l}^{a}=\left(1+\widehat{\varepsilon}_{0}^{m}\right)\left(\widehat{\sigma}_{0}+D\right) H_{i j k l}+\widehat{\sigma} A_{i j k l},
$$

with

$$
\begin{gathered}
D=\left(1+\widehat{\varepsilon}_{0}^{m}\right)\left(\frac{\partial \widehat{\sigma}_{0}}{\partial \widehat{\varepsilon}_{0}^{m}}+\frac{1}{\Delta t} \frac{\partial \widehat{\sigma}_{0}}{\partial \dot{\varepsilon}_{0}^{m}}\right) \\
H_{i j k l}=\widehat{m}_{i} \widehat{m}_{j} \widehat{m}_{k} \widehat{m}_{l}, \\
A_{i j k l}=\frac{1}{2}\left(\delta_{i k} \widehat{m}_{j}+\delta_{j k} \widehat{m}_{i}\right) \widehat{m}_{l}+\frac{1}{2}\left(\delta_{i l} \widehat{m}_{j}+\delta_{j l} \widehat{m}_{i}\right) \widehat{m}_{k}-2 H_{i j k l} .
\end{gathered}
$$

In equations 47-51 all quantities are evaluated at the end of the increment under consideration, i.e., at $t=t_{n+1}$. It is noted that the fourth-order tensor $\widehat{\mathcal{C}}$ has both the "minor" and "major" symmetries, i.e.,

$$
\widehat{\mathcal{C}}_{i j k l}=\widehat{\mathcal{C}}_{j i k l}=\widehat{\mathcal{C}}_{i j l k}=\widehat{\mathcal{C}}_{k l i j}
$$




\section{References}

ABAQUS, 2009. Dassault Systems Abaqus. ABAQUS/Standard Version 6.9, Simulia Corp.

Agoras, M., Lopez-Pamies, O., Castaneda, P.P., 2009. A general hyperelastic model for incompressible fiber-reinforced elastomers. J. Mech. Phys. Solids 57, 268-286.

Bigoni, Z., Serkov, S.K., Valentini, M., Movchan, A.B., 1998. Asymptotic models of dilute composites with imperfectly bonded inclusions. Int. J. Solids and Structures 35, 3239-3258.

Bleiler, C., Castaneda, P.P., Röhrle, O., 2015. Towards effective mechanical properties of skeletal muscle tissue via homogenisation. Proc. Appl. Math. Mech. 15, 83-84.

Blemker, S.S., Pinsky, P.M., Delp, S.L., 2005. A 3D model of muscle reveals the causes of nonuniform strains in the biceps brachii. J. Biomech. 38, 657-665.

Böl, M., Reese, S., 2008. Micromechanical modelling of skeletal muscles based on the finite element method. Comput. Methods Biomech. Biomed. Engin. 11, 489-504.

Böl, M., Weikert, R., Weichert, C., 2011. A coupled electromechanical model for the excitation-dependent contraction of skeletal muscle. J. Mech. Behav. Biomed. Mater. 4, 1299-1310.

Ceelen, K.K., Oomens, C.W.J., Baaijens, F.P.T., 2008. Microstructural analysis of deformation-induced hypoxic damage in skeletal muscle. Biomech. Model. Mechanobiol. 7, 277-284.

Chen, H., Liu, Y., Zhao, X., Lanir, Y., Kassab, G.S., 2011. A micromechanics finite-strain constitutive model of fibrous tissue. J. Mech. Phys. Solids 59, 1823-1837.

deBotton, G., Hariton, I., Socolsky, E.A., 2006. Neo-Hookean fiber-reinforced composites in finite elasticity. J. Mech. Phys. Solids 54, 533-559.

deBotton, G., Oren, T., 2013 Analytical and numerical analyses of the micromechanics of soft fibrous connective tissues. Biomech. Model. Mechanobiol. 12, 151-166.

Destrade, M., Horgan, C.O., Murphy, J.G., 2014. Dominant negative Poynting effect in simple shearing of soft tissues. J. Eng. Math. 95, 87-98.

Ehret, A.E., Böl, M., Itskov, M., 2011. A continuum constitutive model for the active behaviour of skeletal muscle. J. Mech. Phys. Solids 59, 625-636.

Emery, A.E.H., 2002. The muscular dystrophies. Lancet 359, 687-695.

Gao, Y., Wineman, A.S., Waas, A.M., 2008. Mechanics of muscle injury induced by lengthening contraction. Ann. Biomed. Eng. 36, 1615-1623.

Gindre, J., Takaza, M., Moerman, K.M., Simms, C.K., 2013. A structural model of passive skeletal muscle shows two reinforcement processes in resisting deformation. J. Mech. Behav. Biomed. Mater. 22, 84-94.

Gillies, A.R., Lieber, R.L., 2011. Structure and function of the skeletal muscle extracellular matrix. Muscle Nerve 44, 318-331. Hashin, Z., 1990. Thermoelastic properties of fiber composites with imperfect interface. Mechanics of Materials 8, 333-348.

Hashin, Z., 1992. Extremum principles for elastic heterogeneous media with imperfect interfaces and their application to bounding of effective moduli. J. Mechanics and Physics of Solids 40, 767-781.

Hawkins, D., Bey, M., 1994. A comprehensive approach for studying muscle-tendon mechanics. J. Biomech. Eng. 116, 51-55.

Heidlauf, T., Röhrle, O., 2014. A multiscale chemo-electro-mechanical skeletal muscle model to analyze muscle contraction and force generation for different muscle fiber arrangements. Front. Physiol. 5, 498/1-14.

Hernández-Gascón, B., Grasa, J., Calvo, B., Rodríguez, J.F., 2013. A 3D electro-mechanical continuum model for simulating skeletal muscle contraction. J. Theor. Biol. 335, 108-118.

Holzapfel, G.A., Gasser, T.C., Ogden, R.W., 2000. A new constitutive framework for arterial wall mechanics and a comparative study of material models. J. Elasticity $61,1-48$. 
Holzapfel, G.A., Schulze-Bauer, C.A.J., Feigl, G., Regitnig, P., 2005. Single lamellar mechanics of the human lumbar anulus fibrosus. Biomech. Model. Mechanobiol. 3, 125-140.

Holzapfel, G.A., Ogden, R.W., 2015. On the tension-compression switch in soft fibrous solids. Eur. J. Mech. A-Solid 49, $561-569$.

Huijing, P.A., 1999. Muscle as a collagen fiber reinforced composite: a review of force transmission in muscle and whole limb. J. Biomech. 32, 329-345.

Janmey, P.A., McCormick, M.E., Rammensee, S., Leight, J.L., Georges, P.C., MacKintosh, F.C., 2007 Negative normal stress in semiflexible biopolymer gels. Nat. Mater. 6, 48-51.

Javili, A., Chatzigeorgiou, G., Steinmann, P., 2013. Computational homogenization in magneto-mechanics. Int. J. Solids. Struct. 50, 4197-4216.

Johansson, T., Meier, P., Blickhan, R., 2000. A finite-element model for the mechanical analysis of skeletal muscles. J. Theor. Biol. 206, 131-149.

Jozsa, L., Thöring, J., Järvinen, M., Kannus, P., Lehto, M., Kvist, M., 1988. Quantitative alterations in intramuscular connective tissue following immobilization: an experimental study in the rat calf muscles. Exp. Mol. Pathol. 49, $267-278$.

Kjær, M., 2004. Role of extracellular matrix in adaptation of tendon and skeletal muscle to mechanical loading. Physiol. Rev. 84, 649-698.

Lemos, R.R., Epstein, M., Herzog, W., Wyvill, B., 2004. A framework for structured modeling of skeletal muscle. Comput. Methods Biomech. Biomed. Engin. 7, 305-317.

Liang, Y., McMeeking, R.M., Evans, A.G., 2006. A finite element simulation scheme for biological muscular hydrostats. J. Theor. Biol. 242, 142-150.

Lieber, R.L., Runesson, E., Einarsson, F., Fridén, J., 2003. Inferior mechanical properties of spastic muscle bundles due to hypertrophic but compromised extracellular matrix material. Muscle Nerve 28, 464-471.

López Jímenez, F., 2014. Modeling of soft composites under three-dimensional loading. Compos. Part B Eng. 59, 173-180.

Lopez-Pamies, O., Idiart, M.I., 2010. Fiber-reinforced hyperelastic solids: a realizable homogenization constitutive theory. J. Eng. Math. 68, 57-83.

Lopez-Pamies, O., Goudarzi, T., Danas, K., 2013. The nonlinear elastic response of suspensions of rigid inclusions in rubber. II. A simple explicit approximation for finite-concentration suspensions. J. Mech. Phys. Solids 61, 19-37.

Mann, C.J., Perdiguero, E., Kharraz, Y., Aguilar, S., Pessina, P., Serrano, A.L., Muñoz-Cánoves, P., 2011 Aberrant repair and fibrosis development in skeletal muscle. Skelet. Muscle 1, 1-20.

Martins, J.A.C., Pires, E.B., Salvado, R., Dinis, P.B., 1998. A numerical model of passive and active behavior of skeletal muscles. Comput. Methods Appl. Mech. Eng. 151, 419-433.

Mbiakop, A., Constantinescu, A., Danas, K., 2015. An analytical model for porous single crystals with ellipsoidal voids. J. Mech. Phys. Solids 84, 436-467.

Meyer, G.A., Lieber, R.L., 2011. Elucidation of extracellular matrix mechanics from muscle fibers and fiber bundles. J. Biomech. 44, 771-773.

Michel, J.C., Moulinec, H., Suquet, P., 1999. Effective properties of composite material with periodic microstructure: a computational approach. Comput. Methods Appl. Mech. Eng. 172, 109-143.

Purslow, P.P., 1989 Strain-induced reorientation of an intramuscular connective tissue network: implications for passive muscle elasticity. J. Biomech. 22, 21-31.

Purslow, P.P., Trotter, J.A., 1994. The morphology and mechanical properties endomysium in series-fibred muscles: variations with muscle length. J. Muscle Res. Cell. Motil. 15, 299-308.

Röhrle, O., Pullan, A.J., 2007. Three-dimensional finite element modelling of muscle forces during mastication. J. Biomech. 40, 3363-3372. 
Sharafi, B., Blemker, S.S., 2010. A micromechanical model of skeletal muscle to explore the effects of fiber and fascicle geometry. J. Biomech. 43, 3207-3213.

Sharafi, B., Blemker, S.S., 2011. A mathematical model of force transmission from intrafascicularly terminating muscle fibers. J. Biomech. 44, 2031-2039.

Smith, L.R., Lee, K.S., Ward, S.R., Chambers, H.G., Lieber, R.L., 2011. Hamstring contractures in children with spastic cerebral palsy result from a stiffer extracellular matrix and increased in vivo sarcomere length. J. Physiol. 589, 2625-2639.

Spring, D.W., Paulino, G.H., 2015. Computational homogenization of the debonding of particle reinforced composites: The role of interphases in interfaces. Comp. Mater. Sci. 109, 209-224.

Spyrou, L.A., 2009. Muscle and tendon tissues: constitutive modeling, numerical implementation and applications. Dissertation, University of Thessaly.

Spyrou, L.A., Aravas, N., 2011. Muscle and tendon tissues: constitutive modeling and computational issues. J. Appl. Mech. 78, 041015/1-10.

Spyrou, L.A., Aravas, N., 2012. Muscle-driven finite element simulation of human foot movements. Comput. Methods Biomech. Biomed. Engin. 15, 925-934.

Suchocki, C., 2011. A finite element implementation of Knowles stored-energy function: theory, coding and applications. Arch. Mech. Eng. 58, 319-346.

Thacker, B.E., Tomiya, A., Hulst, J.B., Suzuki, K.P., Bremner, S.N., Gastwirt, R.F., Greaser, M.L., Lieber, R.L., Ward, S.R., 2012. Passive mechanical properties and related proteins change with botulinum neurotoxin A injection of normal skeletal muscle. J. Orthop. Res. 30, 497-502.

Tkachuk, M., Linder, C., 2012. The maximal advance path constraint for the homogenization of materials with random network microstructure. Philos. Mag. 92, 2779-2808.

Trotter, J.A., Richmond, F.J.R., Purslow, P.P., 1995. Functional morphology and motor control of series-fibered muscles. Exerc. Sport Sci. Rev. 23, 167-214.

Van Leeuwen, J.L., Kier, W.M., 1997. Functional design of tentacles in squid: linking sarcomere ultrastructure to gross morphological dynamics. Philos. Trans. R. Soc. Lond. B Biol. Sci. 352, 551-571.

Van Loocke, M., Lyons, C.G., Simms, C.K., 2006. A validated model of passive muscle in compression. J. Biomech. 39, 2999-3009.

Virgilio, K.M., Martin, K.S., Peirce, S.M., Blemker, S.S., 2015. Multiscale models of skeletal muscle reveal the complex effects of muscular dystrophy on tissue mechanics and damage susceptibility. Interface Focus 5, 20140080/1-10.

Würkner, M., Berger, H., Gabbert, U., 2013. Numerical study of effective elastic properties of fiber reinforced composites with rhombic cell arrangements and imperfect interface. Int. J. Eng. Sci. 63, 1-9.

Zajac, F.E., 1989. Muscle and tendon: properties, models, scaling and application to biomechanics and motor control. Crit. Rev. Biomed. Eng. 17, 359-411. 